\title{
ELECTRON CORRELATION IN METALS
}

\author{
K. YAMADA \\ Department of Physics, Kyoto University, Japan
}




\section{Contents}

Preface

1 Fermi gas

1.1 Metals

page vii

1.2 Free Fermi gas 2

1.3 Electronic specific heat and Pauli susceptibility 4

1.4 Many-body effect of electron gas 7

$\begin{array}{ll}1.5 \text { Exchange energy } & 10\end{array}$

1.6 Screening effect 13

1.7 Plasma oscillation $\quad 16$

1.8 Ground state energy 18

1.9 Wigner crystal 20

2 Fermi liquid theory $\quad 22$

2.1 Principle of continuity 22

2.2 Landau's Fermi liquid theory 25

3 Anderson's orthogonality theorem 29

3.1 Friedel sum rule 29

3.2 Orthogonality theorem on local perturbation 33

3.3 Photoemission in metals and the orthogonality theorem 36

3.4 Diffusion of charged particles in metals 41

4 s- $d$ Hamiltonian and Kondo effect $\quad 49$

4.1 Spin susceptibility of conduction electrons 49

$4.2 s-d$ Exchange interaction and spin polarization $\quad 51$

4.3 Kondo effect 54

4.4 Ground state of dilute magnetic alloy system 60

4.5 Scaling law of the $s-d$ system 63

$\begin{array}{ll}4.6 \text { Wilson's theory } & 67\end{array}$

4.7 Fermi liquid theory by Nozières 68 
5 Anderson Hamiltonian $\quad 72$

$\begin{array}{ll}5.1 \text { Hartree-Fock approximation } & 72\end{array}$

5.2 Perturbation expansion with respect to $V_{k d} \quad 76$

$\begin{array}{ll}5.3 \text { Green's function } & 79\end{array}$

5.4 Perturbation expansion with respect to $U \quad 87$

5.5 Exact solution of the Anderson Hamiltonian 99

6 Hubbard Hamiltonian 103

$\begin{array}{ll}6.1 \text { Basic properties } & 103\end{array}$

$\begin{array}{ll}6.2 \text { Theory of electron correlation } & 106\end{array}$

6.3 Infinite-dimensional Hubbard Hamiltonian 112

$\begin{array}{ll}\text { 6.4 Mott transition } & 117\end{array}$

6.5 One-dimensional Hubbard model 119

$\begin{array}{ll}\text { 6.6 Ferromagnetism of transition metals } & 120\end{array}$

6.7 Superconductivity in the Hubbard Hamiltonian 121

7 Fermi liquid theory of strongly correlated electron systems 122

$\begin{array}{ll}7.1 \text { Heavy fermion systems } & 122\end{array}$

$\begin{array}{ll}7.2 \text { Heavy fermions } & 123\end{array}$

$\begin{array}{ll}7.3 \text { Kondo temperature in crystalline fields } & 124\end{array}$

$\begin{array}{ll}7.4 \text { Fermi liquid theory on heavy fermion systems } & 127\end{array}$

$\begin{array}{ll}7.5 \text { Spin fluctuation and Fermi Liquid } & 141\end{array}$

8 Transport theory based on Fermi liquid theory 147

$\begin{array}{ll}8.1 \text { Conductivity } & 147\end{array}$

$\begin{array}{ll}8.2 \text { Optical conductivity } & 148\end{array}$

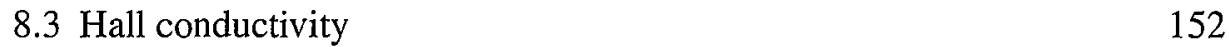

8.4 Cyclotron resonance $\quad 153$

$\begin{array}{ll}\text { 8.5 Magnetoresistance } & 157\end{array}$

$\begin{array}{ll}8.6 \text { Concluding remarks } & 158\end{array}$

9 Superconductivity in strongly correlated electron systems $\quad 159$

$\begin{array}{ll}9.1 \text { Cuprate high-temperature superconductors } & 159\end{array}$

9.2 Anisotropic superconductivity due to coulomb repulsion 172

$\begin{array}{ll}9.3 \text { Electron-doped cuprates } & 206\end{array}$

$\begin{array}{ll}\text { 9.4 Triplet superconductor } \mathrm{Sr}_{2} \mathrm{RuO}_{4} & 210\end{array}$

$\begin{array}{ll}9.5 \text { Conclusion } & 218\end{array}$

Appendix A. Feynman relation $\quad 223$

$\begin{array}{ll}\text { Appendix B. Second quantization } & 224\end{array}$

Appendix $C$. Interaction representation and thermal

$\begin{array}{ll}\text { Green's function } & 226\end{array}$

Appendix D. Linear response theory 233

Appendix E. Transport equation derived by Éliashberg 238

$\begin{array}{ll}\text { Index } & 243\end{array}$ 